\title{
A thoughtful approach to brain tumours
}

\author{
Manuel Deprez \\ Laboratoire de Neuropathologie, Tour de Pathologie, B-35, CHU-Liège, Sart Tilman, B-4000, Belgium
}

Brain Tumors: an Encylopedic Approach, Second Edition

Andrew H Kaye, Edward R Laws Jr. London: Churchill Livingstone, 2001. Pp 1080. £175.00. ISBN 0443064261.

Brain Tumours is a serious book, more than 1000 pages long, with 50 chapters, and the efforts of nearly 100 contributors. The editors begin by reminding us that surgery has always had a central role in the management of brain tumours. However, surgical resection is often incomplete because of diffuse infiltration and the anatomical limits imposed by the surgical approach, or by proximity to the eloquent cortex. Incomplete resection undoubtedly contributes to tumour progression or recurrence, and the means to solve this dilemma lie in the collaborative effort of various medical and scientific disciplines. Such a multidisciplinary approach is all very well, but it requires open lines of communication between specialties that often differ substantially in their conceptual and technical approaches. Two editors, who apparently wish to obtain the best treatment combination for any given patient, have bravely assembled a vast quantity of knowledge usually dispersed in multiple specialties and subspecialties in one hefty book. The experts' monologues are elucidated here by juxtaposition. Unlike the alphabetical order of subject matter used in most modern encyclopaedias, these editors have justifiably preferred an organisation by theme, grouped in two principal sections: basic principles and specific brain tumours.

The first section deals with the biology and the morphology of brain tumours, then takes on diagnostic techniques and treatments. The first chapter gives an historic overview of the treatment of brain tumours, unduly prolonged in the second chapter, which describes the target cells of neoplastic transformation in the developing brain, and for which the most recent reference dates from 1993. By contrast, chapter 3 is motivated by the task of updating the classification of brain tumours in line with WHO's 2000 revision. However, this interesting chapter would have been better placed 120 pages further on, next to chapter 9, on tumour histology. Such initial confusion is happily dissipated by a succession of 20 chapters that are remarkably well written, illustrated, and updated. Overall, this book provides a solid basis for a critical read of the second section, in which each specific histological type of tumour is examined in its biological, epidemiological, diagnostic, and therapeutic aspects, echoing the principles illustrated in the first section, and providing evidence for the character of cyclical teaching contained in the word encyclopaedia itself.

In the tradition of the systematic encyclopaedia, each author contributes freely his expertise in a precise domain. Different interpretations of the same data, and divergent points of view, thus crop up as often in such reading as they do at conferences. Particularly in matters of diagnosis or treatment, the pioneering enthusiasm of some is tempered by the acquired or inherited prudence of others. For example, although a consensus exists about the interest of metabolic and functional imaging techniques for guiding biopsies or tumour resection, certain neurosurgeons differ in the validity that they accord these techniques elsewhere. Compare the quotes below on making the difficult differential diagnosis between a glioma's recurrence and radiation necrosis; also evident are the differing views between researchers in immunology and neurosurgeons on the interpretation of the first results obtained with immunotherapy (panel).

Such divergence in these pages is the exception rather than the rule, and underscores the high standards of the editors and contributors. The editors do not specify to whom this book is addressed, but even the number of neurosurgeons among the collaborators should not restrict this work to an exclusively medical and surgical audience. As a neuropathologist, I read this volume with great interest, and I recommend it to all those who are interested in brain tumours and wish to integrate their highly specialised viewpoints with a broader perspective. In providing the basis of mutual understanding and respect between specialists with very different horizons, this book remains faithful to the spirit of the first encyclopaedists. 


\section{Different interpretations}

\section{Glioma's recurrence or radiation necrosis?}

"Perhaps most potentially worthwhile is the application of MRSI [Magnetic Resonance Spectroscopic Imaging] to differentiate between recurrent glioma and radiation change in diagnostically difficult cases."

"Although specificity in differentiating tumor from radiation necrosis of up to $100 \%$ has been claimed, in many cases these studies are inconclusive and the diagnosis is revealed either by the clinical course or by analysis of a pathology specimen."

\section{Interpreting first results with immunotherapy}

"Encouraging results have been observed in the first clinical trials, suggesting that the concept of successful treatment with 'educated' immune cells is not an unrealizable aim." "That immunotherapy has failed to improve the survival of patients with malignant brain tumors is not surprising given its modest record in the treatment of solid tumors outside the nervous system . . . Unfortunately, most immunologic agents suffer from either low activity or low potency ... In conclusion, immunotherapy is unlikely to succeed as single modality treatment; however, it would appear to have great potential as a carrier and targeting technology in combination with other agents." 